\title{
Nuclear reprogramming and the cancer
}

\section{genome}

\author{
Three disciplines - cancer genomics, functional analysis of cancer cells and nuclear reprogramming-have come \\ together to focus on the basic biology of cell commitment in our Nature Conference with the Ludwig Institute for \\ Cancer Research held 25-27 September in Oxford, UK.
}

$\mathrm{H}$ undreds of thousands of genetic elements in a cell's genome could theoretically in combination specify a vast number of cell states. Cells in real multicellular organisms, however, are constrained to adopt a much smaller number of states: a few hundred histologically distinct cell types are descended from three embryonic lineages in the human body. Genetic elements, their chromatin marks and interacting transcription factors form the basis of each cell type's characteristic transcriptional state and confer parallel properties of attractor networking (whereby cell states resist perturbation) and lineage memory (whereby cells retain restrictions on pluripotency and gene expression from earlier points in their developmental trajectory). Characterization of the signals that initiate transitions between cell states has provided rich mechanistic insights in biomedical research. In contrast, the complex cellular system that undergoes state transitions is less well understood, as it is a much larger search space, although it is now much more accessible thanks to years of systematic application of genomics to human cells and developmental models.

Thousands of cancer genomes and their original constitutional genomes have now been read for dozens of tumor types (Science 339, 1546-1558, 2013), and, through the use of genome-wide tools of bisulfite sequencing, array genotyping, RNA sequencing and chromatin immunoprecipitation, the epigenetic adaptations of the cancer genome and its reprogrammed derivatives can be separated from the more readily accessible mutational component.

Experimental reprogramming of cancer cells has demonstrated the range of proliferative and non-proliferative fates accessible to the cancer genome, despite the acquisition of oncogenic mutations and loss of tumor suppressors. For example, a melanoma nucleus from a mouse, injected into an oocyte, was reprogrammed to totipotency (Genes Dev. 18,1875-1885, 2004), and induced pluripotent stem cells (iPSCs) have been generated from chronic myeloid leukemia cells (Blood 115, 4039-4042, 2010). These classic results suggest that it is the rarely mutated but commonly-and globally_dysregulated epigenomes of cancer cells that will yield the greatest insights when we compare them with the epigenomes of experimentally reprogrammed cells (Nat. Rev. Cancer 13, 497-510, 2013). If so, this is encouragement for a synthesis between cancer genome projects and regenerative medicine, as important aims of reprogramming cells are to avoid inducing mutations and to understand the epigenetics underlying cell fate.
Reprogramming entails several coordinated changes that occur at different times in different protocols. In contrast to the comprehensive reorganization that an introduced nucleus experiences when it is exposed to the cytoplasm of an oocyte or early embryo (Nat. Rev. Mol. Cell Biol. 12, 453-459, 2011), iPSC generation remains a stochastic process. Induced pluripotency protocols are only partly deterministic, as reprogramming factors can only bring about expression of pluripotency genes in those few cells that have sporadically become responsive. The frequency of reprogramming can be increased and the process made less dependent on signals from the culture environment by adding global epigenetic intervention, for example, by swapping regions of heterochromatin for euchromatin via demethylation of histone $\mathrm{H} 3$ at lysine 9 (Nat. Genet. 45, 34-42, 2013).

Stem cells and cancer cells share the property of self-renewal. Some of the Yamanaka factors used to induce pluripotency in somatic cells (Cell 126, 663-676, 2006) also have important roles in the genesis of some tumor types (for example, MYC (Nat. Rev. Cancer 11, 268-277, 2011) and SOX2 (Nat. Genet. 44, 1111-1116, 2012 and Nat. Genet. 41, $1238-1242,2009)$ ), and it is clearly imperative that regenerative medicine gain full control over reprogrammed cell fates. The biology of cell commitment is relevant not only to regenerative medicine but also to understanding stem cell properties of cancers and in developing cancer treatment strategies. At tumor initiation, mutations (or epigenetic alterations) in normal stem cell populations can give rise to a higher probability of evolving a characteristic tumor than if the mutations arose in another cell type (for examples, see Nature 468, 1095-1099, 2010 and Sci. Transl. Med. 4, 149ra118, 2012). In tumor progression, chromatin alterations in cancer cells can remove the epigenetic barrier between the cancer stem cell and cancer cell fates, conferring adaptive proliferative and malignant potential (Nature 488, 462-463, 2012 and Cell 154, 61-74, 2013).

Currently, most of the biology of iPSCs and cancer cells is described in very different terms and is practiced in very different laboratories. But the good news is that the three disciplines of nuclear reprogramming, cancer genomics and functional analysis of cancer cells are eminently complementary strategies that, when appropriately braided together, have the potential to bring important insights into the key properties of multicellular life-potency and commitment. 